15.1;05.5;11.4

\title{
Сверхпроводящий источник шума для сверхнизких температур
}

\author{
(C) T.М. Ким ${ }^{1}$, С.В. Шитов ${ }^{1,2, q}$ \\ ${ }^{1}$ Национальный исследовательский технологический университет „МИСиС“, Москва, Россия \\ ${ }^{2}$ Институт радиотехники и электроники им. В.А. Котельникова РАН, Москва, Россия \\ E-mail: kim.tatyana.mail@gmail.com \\ ฯ E-mail: sergey3e@gmail.com
}

Поступило в Редакцию 1 июня 2021 г.

В окончательной редакции 21 июля 2021 г.

Принято к публикации 12 сентября 2021 г.

\begin{abstract}
Сверхпроводящий источник шума работает при температурах $<300 \mathrm{mK}$ и содержит микромостик из гафния и сверхпроводящий туннельный переход из алюминия, включенные в общий копланарный волновод. Микромостик согласован с планарной антенной и реализует функцию оптического черного тела на частотах 600-700 GHz. Копланарная линия является выходом черного тела в диапазоне $1-2 \mathrm{GHz}$. Температура микромостика устанавливается в диапазоне $0.4-9 \mathrm{~K}$ и калибруется с использованием дробового шума туннельного перехода. Модуляция температуры каждого из источников контролируется независимо с помощью постоянного тока посредством перевода из сверхпроводящего в нормальное состояние с характерными временами $<0.1 \mathrm{~ms}$ и тепловыделением $\sim 1 \mu \mathrm{W}$.
\end{abstract}

Ключевые слова: сверхпроводящий микромостик, сверхпроводящий туннельный переход, термодинамический шум, дробовой шум, шумовая термометрия.

DOI: 10.21883/PJTF.2021.24.51791.18897

Шумовые измерения при сверхнизких температурах (30-300 mK) необходимы для калибровки сверхчувствительных тепловых детекторов для исследований космоса в терагерцевом $(\mathrm{THz})$ диапазоне [1] и усилителей в цепях квантовой обработки информации в гигагерцевом $(\mathrm{GHz})$ диапазоне [2]. Существующие методы чернотельного излучателя (ЧТИ) используют постоянный [3] или импульсный [4] нагрев ЧТИ мощностью $\sim 1 \mathrm{~mW}$, что может быть критичным для рефрижератора растворения с мощностью охлаждения $\sim 100 \mu \mathrm{W}$. В работе [5] ЧТИ [4] охарактеризован как структурный аналог композитного болометра размером $\sim 10 \times 10 \mathrm{~mm}$ массой $\sim 0.1 \mathrm{~g}$, в котором импульсы напряжения $\sim 10 \mathrm{~V}$ вызывают нагрев пленочного поглотителя с фронтом $\sim 1 \mathrm{~ms}$. Это позволяет калибровать чувствительность и скорость реакции изображающих матриц [5]. Однако эффективное время цикла нагрев-охлаждение такого источника составляет $\tau \sim 2 \mathrm{~s}$, что затрудняет реализацию радиометра с частотами модуляции $f_{m}>1 \mathrm{~Hz}$ : при $f_{m} \sim 10 \mathrm{~Hz}$ температурная амплитуда ЧТИ снижается $\sim\left(\tau f_{m}\right)^{-1}$. $\mathrm{B}$ настоящей работе анализируются электрофизическая модель и конструкция интегральной схемы шумового калибратора, преимуществами которого являются малая тепловая мощность $(\sim 1 \mu \mathrm{W})$ и короткое время тепловой релаксации $(\tau<0.1 \mathrm{~ms})$. Предлагаемый нами ЧТИ также напоминает болометр, но в его основе лежат резистивный микромостик и планарная THz-антенна. Диапазон шумовых температур калибратора 0.4-9K оптимален для исследования THz-детекторов, работающих в условиях космического фона с температурой $T_{b g} \approx 2.7 \mathrm{~K}$. Физическую температуру $T$ пленочного резистора можно задавать либо нагревом подложки, либо током, разогревающим пленку относительно подложки. Первый способ реализован в ЧТИ [4], второй требует определения локальной температуры пленки, что невозможно ни при использовании стандартных криогенных термометров [6], ни при использовании интегральных структур типа [4]. Предлагается определять температуру микрорезистора, сравнив его шум на GHz-частотах с дробовым шумом туннельного перехода типа SIS (superconductor-insulator-superconductor) [7,8], и использовать ее для вычисления термодинамического шума на $\mathrm{THz}$-частотах, что является развитием методов шумовой термометрии [9]. Принципиальная схема устройства представлена на рис. 1. Два источника шума включены последовательно в общую электрическую цепь на одном чипе. К выходу чипа подключается референсный измеритель мощности - охлаждаемый детектор или малошумящий охлаждаемый усилитель в радиометрическом режиме с мгновенной полосой $1 \mathrm{GHz}$ и шумовой температурой $\sim 10 \mathrm{~K}$. Сверхпроводящее и резистивное состояния микромостика и SIS-перехода позволяют: 1) выключить любой из них, переведя в сверхпроводящее состояние; 2) передать без потерь сигнал от выбранного источника; 3) управлять независимо уровнем шума с помощью тока смещения в широком диапазоне частот (до $\sim 100 \mathrm{kHz}$ ); 4) использовать критические температуры материалов как референсные параметры. Мощность шума $P_{n}$ идеального резистора может быть описана формулой Планка в неограниченном диапазоне частот $\Delta f$. В пределе низких частот или больших температур $\left(h f \ll k_{\mathrm{B}} T\right.$, где $h-$ постоянная Планка, $f-$ частота, $k_{\mathrm{B}}-$ константа Больцмана) мощность шума, переданная в нагрузку, $P_{n}=k_{\mathrm{B}} T \Delta f$. 


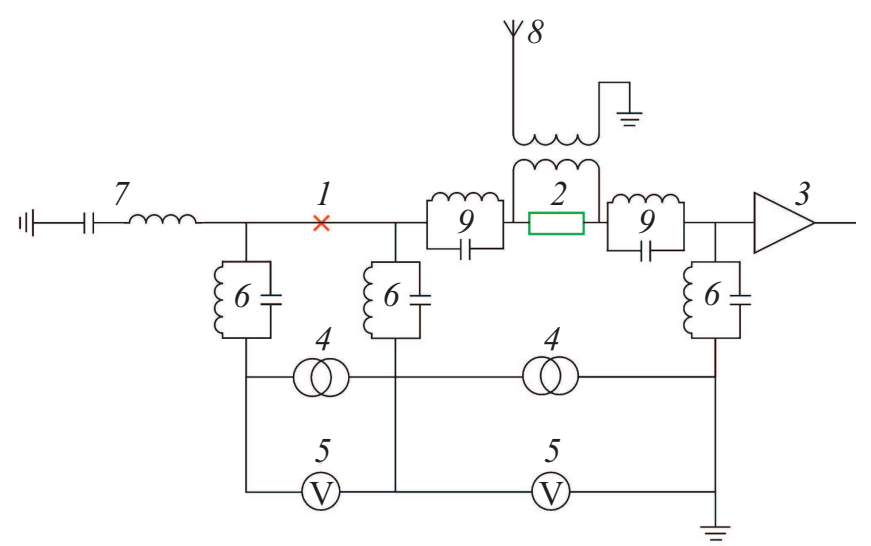

Рис. 1. Принципиальная схема устройства. 1 - SIS-переход, 2 - сверхпроводящий микромостик, 3 - референсный измеритель мощности, 4 - источники тока, 5 - измерители напряжения, 6 - полосно-заграждающие фильтры диапазона $1-2 \mathrm{GHz}, 7$ - полосно-пропускающий фильтр $1-2 \mathrm{GHz}, 8-$ двухщелевая THz-антенна, 9 - полосно-заграждающие фильтры антенны 600-700 GHz.

При условии $e V \gg k_{\mathrm{B}} T \gg h f$ (где $e-$ заряд электрона, $V$ - напряжение) в SIS-переходе доминирует дробовой шум, который определяется током $I$. Квадрат напряжения дробового шума $\left\langle V_{\text {shot }}^{2}\right\rangle=2 e I R_{n}^{2} \Delta f$, где $R_{n}-$ нормальное сопротивление, откуда согласованная мощность $P_{s h o t}=e I R_{n} \Delta f / 2$. Эквивалентная температура дробового шума $T_{s h o t}=P_{s h o t} / 2 k_{\mathrm{B}} \Delta f$ пропорциональна $I$ на линейном участке вольт-амперной характеристики (BAX) (рис. 2,a), минимальное значение $T_{\text {shot } \min }=e V_{g} / 2 k_{\mathrm{B}}$. Для SIS-перехода из алюминия $V_{g}=340-360 \mu \mathrm{V}$, тепловыделение $\sim 2 \mathrm{nW}$ и $T_{\text {shot } \min } \approx 2 \mathrm{~K}$. Частотный диапазон $\Delta f_{\text {SIS }}$ дробового шума SIS-перехода площадью $10 \mu \mathrm{m}^{2}$ не превышает $50 \mathrm{GHz}$ и определяется условием $2 \pi \Delta f_{\mathrm{SIS}} R_{n} C_{\mathrm{SIS}}<1$, где $C_{\mathrm{SIS}}-$ емкость SIS-перехода.
Кроме прямого сравнения шумов можно использовать метод теплового гистерезиса ВАХ микромостика [10] (рис. $2, b)$. Превышение критического тока $I_{c}$ приводит к скачку напряжения, и микромостик становится резистором с сопротивлением $R_{n}$, разогретым до температуры $T$, которая заведомо превышает температуру подложки $T_{0}$. Обратный переход в сверхпроводящее состояние реализуется при токе возврата $I_{r}<I_{c}$, при этом $T$ снижается до критического значения $T_{c}$, а разогрев - до $P_{r}=I_{r}^{2} R_{n}$. При $T<1 \mathrm{~K}$ необходимо учитывать эффект электронного газа [11] и в качестве флуктуационной температуры использовать электронную температуру $T_{e}$, которую можно найти, решив уравнение постоянного потока тепла через электрон-фононный и фонон-фононный интерфейсы

$$
P_{e-p h}(T)=\Sigma v\left(T_{e}^{n}-T_{k}^{n}\right)=\frac{S}{A}\left(T_{k}^{4}-T_{0}^{4}\right),
$$

где $T_{k}-$ температура решетки, $\Sigma-$ константа электрон-фононного взаимодействия материала, $v$ объем мостика, $n=5-6, A-$ константа Капицы [12], $S$ - площадь теплового контакта пленки с подложкой. Правая часть уравнения (1) отражает разогрев фононной системы пленки относительно фононной системы подложки; при температурах выше $1 \mathrm{~K} T_{k}-T_{0} \gg T_{e}-T_{k}$. Степень $n$ можно уточнить, измеряя тепловую мощность в самой нижней точке резистивной ветви ВАХ, где $P_{e-p h}(T)=I_{r}\left(T_{0}\right)^{2} R_{n}$. В этой точке рассчитанный шум микромостика должен соответствовать дробовому шуму: $T_{e} \approx T_{\text {shot }}$. Спектр шума мостика с электронным газом можно считать термодинамическим до частот, ограниченных эффективностью андреевских зеркал [13] на границе мостик-сверхпроводящие электроды из ниобия, т. е. до $~ 750 \mathrm{GHz}$. ЧТИ предназначен для использования в двух частотных диапазонах $(1-2$ и $600-700 \mathrm{GHz})$
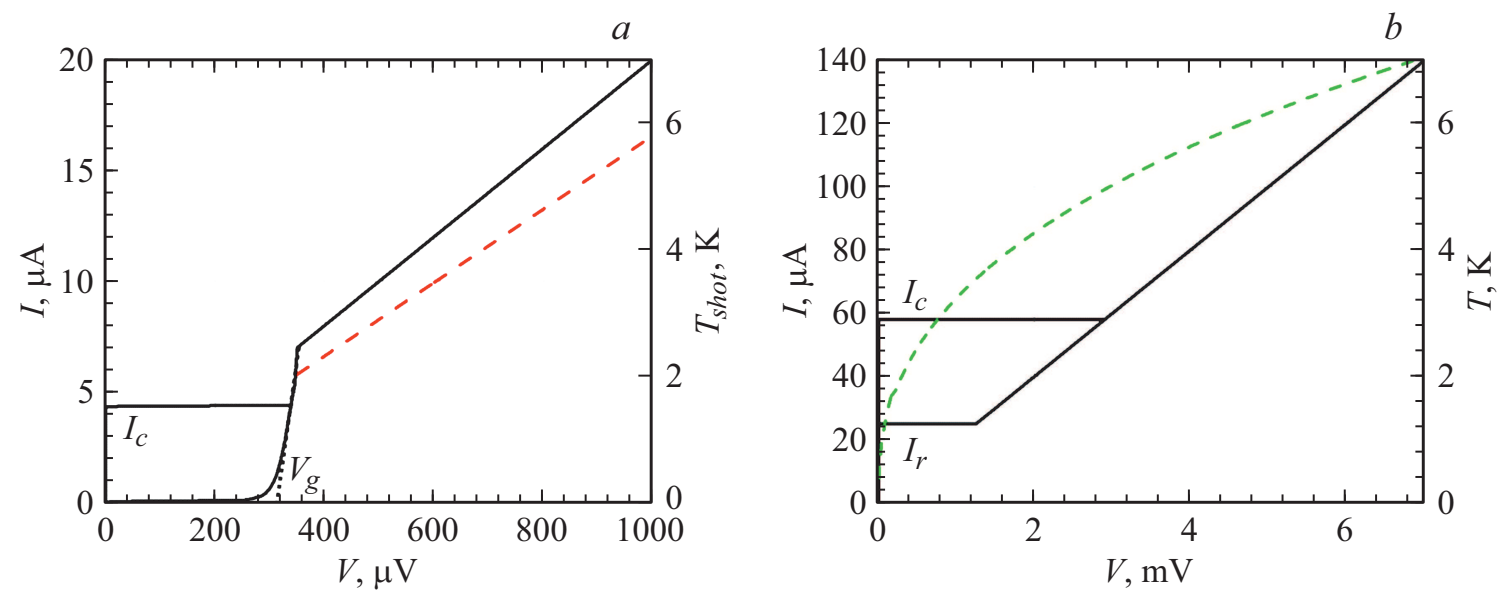

Рис. 2. Характеристики источников шума. $a-\mathrm{BAX}$ туннельного перехода типа SIS (сплошная линия) и шумовая температура $T_{\text {shot }}$, рассчитанная выше щелевого напряжения $V_{g}$ (штриховая линия); $b$ - гистерезисная ВАХ сверхпроводящего микромостика (сплошная линия) и физическая температура $T$, рассчитанная с учетом сильной температурной зависимости теплоотвода (штриховая линия). 


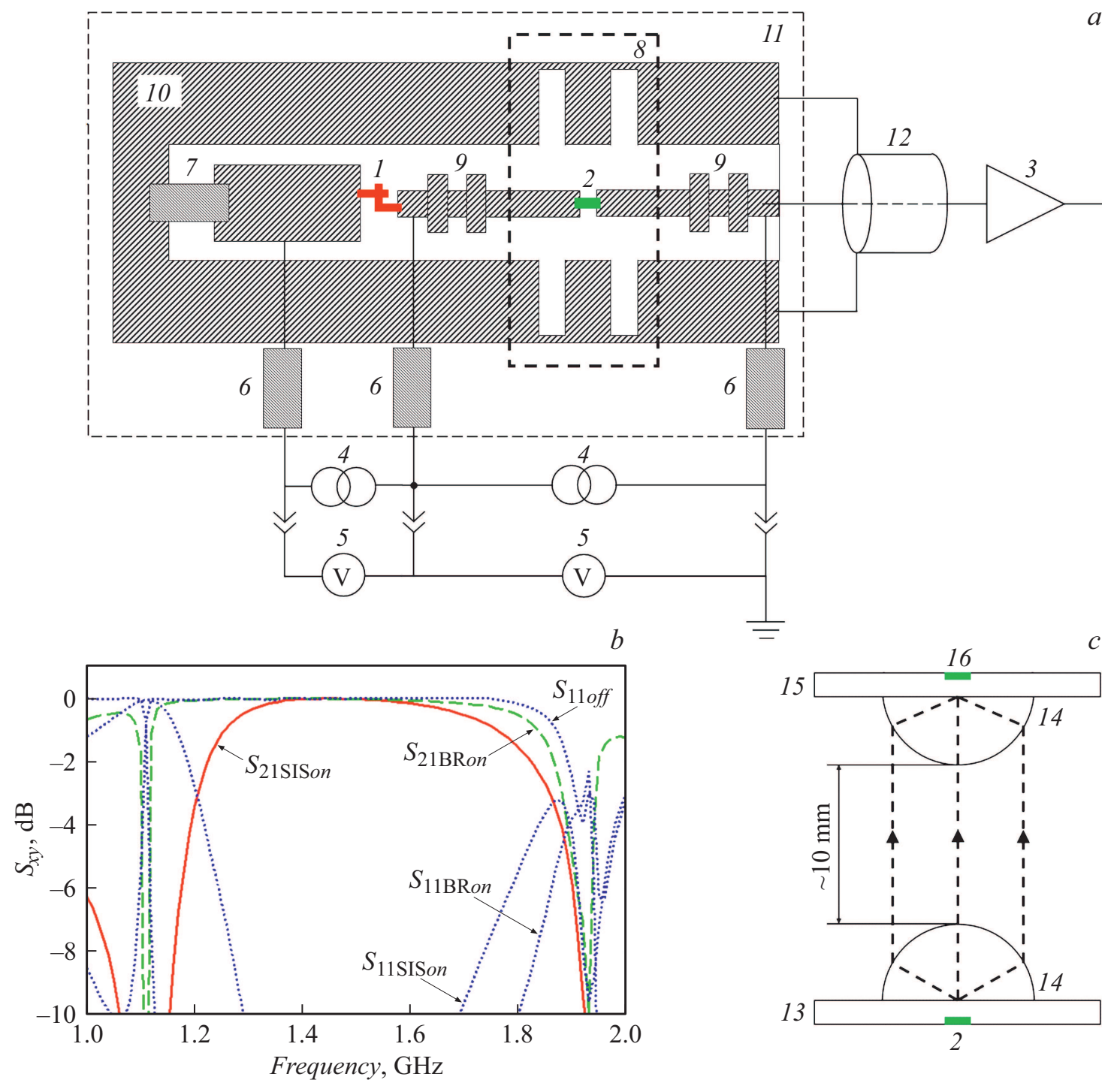

Рис. 3. Конструкция устройства. $a$ - упрощенная топология чипа и внешние цепи; области металлизации чипа заштрихованы. $1-9$ - то же, что на рис. $1,10-$ копланарный волновод, $11-$ диэлектрическая подложка, $12-$ коаксиальный кабель. $b-$ рассчитанные [14] параметры экспериментального чипа: коэффициент передачи сигнала с SIS-перехода $\left(S_{21 \mathrm{SIS} o n}\right)$, коэффициент передачи с микромостика $\left(S_{21 \mathrm{BR} o n}\right)$, коэффициент отражения чипа при выключенных $\left(S_{11 o f f}\right)$ и включенных $\left(S_{11 \mathrm{SISon}}, S_{11 \mathrm{BR} o n}\right)$ источниках. $c$ - схема передачи сигнала ЧТИ с применением коллимирующей иммерсионной оптики для проекта $[1]:$ референсный усилитель 3 подключается к чипу 13, на котором расположен излучатель 2; линза 14 формирует коллимированный пучок, который поступает через аналогичную линзу на чип 15 , где расположен тестируемый детектор 16.

для продолжения проектов [1,15]. Диапазон температур микромостика ограничен критическими температурами гафния и ниобия: $T_{c \mathrm{Hf}} \approx 0.4 \mathrm{~K}$ и $T_{c \mathrm{Nb}} \approx 9 \mathrm{~K}$. Упрощенная топология чипа представлена на рис. $3, a$ : SIS-переход и микромостик включены в общую копланарную линию из ниобия на сапфировой подложке. Микромостик согласован одновременно с двухщелевой THz-антенной $[1,15,16]$ и референсным усилителем, подключенным через копланарный волновод в диапазоне $1-2 \mathrm{GHz}$. Полоснозаграждающие фильтры антенны предотвращают утечку сигнала в диапазоне $600-700 \mathrm{GHz}$ и прозрачны в диапазоне $1-2 \mathrm{GHz}$. Источники тока и измерители напряжения подключены через полосно-заграждающие фильтры 1-2 GHz. Электродинамический расчет чипа в среде Cadence AWR [14] подтвердил реализуемость предложенного устройства в диапазоне $1-2 \mathrm{GHz}$ (рис. $3, b$ ). Чип помещается на плоскую поверхность эллиптической иммерсионной линзы (рис. $3, c$ ) так, что антенна находится в ее фокусе, а тестируемый детектор [15], снабженный аналогичной линзовой антенной (ЛА), расположен на удалении $\sim 10 \mathrm{~mm}$ в ближней зоне, где фронт излучения плоский. Такая система ЛА с типичным коэффициентом направленности $>20 \mathrm{~dB}$ не требует промежуточной оптики и обеспечивает высокую помехозащищенность: ослабление внеосевых пучков составит $>16 \mathrm{~dB}$. Потери сигнала определяются дифрак- 
ционным рассеянием на апертуре ЛА и не превышают $1 \mathrm{~dB}(\sim 20 \%)$. Термодинамический фон криостата $0.3 \mathrm{~K}$ характеризуется границей планковского спектра в районе $10 \mathrm{GHz}$, что позволяет пренебречь фотонной нагрузкой THz-детектора. Микрорезистор $50 \Omega$ размером $2 \times 2 \times 0.05 \mu \mathrm{m} \quad$ с теплопроводностью $G=10^{-9} \mathrm{~W} / \mathrm{K}$ разогревается до $T=9 \mathrm{~K}$ мощностью $3 \mu \mathrm{W}$ при смещении $13 \mathrm{mV}$; при температуре калибровки $2 \mathrm{~K}$ мощность резко падает и составляет $2 \mathrm{nW}$ при напряжении $0.3 \mathrm{mV}$. Оценить паразитный нагрев $\Delta T$ подложки из сапфира с удельной теплопроводностью $G_{s}=10^{-2} \mathrm{~W} /(\mathrm{K} \cdot \mathrm{m})$ при $T_{0}=100 \mathrm{mK}[17]$ можно с помощью упрощенной модели распространения тепла в виде изотермической полусферы диаметром $D$. Соотношение $\Delta T(D)=P / G_{s} \pi D$ является результатом интегрирования градиента температуры между источником диаметра $D$ с мощностью $P$ и бесконечно удаленной изотермической полусферой $T_{0}$. Если расположить SIS-переход на расстоянии $2 \mathrm{~mm}$ от мостика, то рассчитанное в этой точке $\Delta T<10 \mathrm{mK}$, и таким нагревом SIS-перехода можно пренебречь. Проведенные оценки показывают, что предлагаемый генератор шума не перегрузит криостат растворения и в принципе может быть интегрирован в составе микросхемы с практическим болометром и/или усилителем. Эксперимент по модуляции в проекте [15] позволил оценить время температурной релаксации микромостика из гафния $<10 \mu \mathrm{s}$, что определяется временем электрон-фононной релаксации. Использование электронного газа в качестве термодинамической среды [18] открывает качественно новые возможности в прецизионных измерениях сверхмалошумящих устройств.

\section{Финансирование работы}

Работа выполнена при финансовой поддержке Российского фонда фундаментальных исследований в рамках научного проекта № 20-37-90094, а также Министерства образования и науки Российской Федерации в рамках Программы повышения конкурентоспособности НИТУ МИСиС в части материаловедения - грант НИТУ МИСиС № К2-2020-016.

\section{Конфликт интересов}

Авторы заявляют, что у них нет конфликта интересов.

\section{Список литературы}

[1] A.V. Merenkov, V.I. Chichkov, A.B. Ermakov, A.V. Ustinov, S.V. Shitov, IEEE Trans. Appl. Supercond., 28 (7), 1 (2018). DOI: 10.1109/TASC.2018.2827981

[2] B.H. Eom, P.K. Day, H.G. LeDuc, J. Zmuidzinas, Nature Phys., 8, 623 (2012). DOI: 10.1038/nphys 2356

[3] A.V. Uvarov, S.V. Shitov, A.N. Vystavkin, Meas. Techn., 53 (9), 1047 (2010). DOI: 10.1007/s11018-010-9617-4
[4] Ph. Abbon, A. Delbart, M. Fesquet, C. Magneville, B. Mazeau, J.-P. Pansart, D. Yvon, L. Dumoulin, S. Marnieros, Ph. Camus, T. Durand, Ch. Hoffmann, Nucl. Instrum. Meth. Phys. Res. A, 575 (3), 412 (2007). DOI: 10.1016/j.nima.2007.02.094

[5] S. Masi, P. de Bernardis, A. Paiella, F. Piacentini, L. Lamagna, A. Coppolecchia, P.A.R. Ade, E.S. Battistelli, M.G. Castellano, I. Colantoni, F. Columbro, G. D'Alessandro, M. De Petris, S. Gordon, C. Magneville, P. Mauskopf, G. Pettinari, G. Pisano, G. Polenta, G. Presta, E. Tommasi, C. Tucker, V. Vdovin, A. Volpe, D. Yvon, J. Cosmol. Astropart. Phys., 2019 (7), 003 (2019). DOI: 10.1088/1475-7516/2019/07/003

[6] https://www.lakeshore.com/products/categories/ temperatureproducts/cryogenic-temperature-sensors

[7] V.Yu. Belitsky, V.P. Koshelets, I.L. Serpuchenko, M.A. Tarasov, L.V. Filippenko, S.V. Shitov, in Proc. of the 20th Eur. Microwave Conf. (IEEE, 1990), vol. 1, p. 816. DOI: 10.1109/EUMA.1990.336144

[8] H. Inoue, T. Noguchi, K. Kohno, J. Phys.: Conf. Ser., 234, 042014 (2010). DOI: 10.1088/1742-6596/234/4/042014

[9] L. Spietz, R.J. Schoelkopf, P. Pari, Appl. Phys. Lett., 89 (18), 183123 (2006). DOI: 10.1063/1.2382736

[10] B.S. Karasik, S.V. Pereverzev, D. Olaya, J. Wei, M.E. Gershenson, A.V. Sergeev, IEEE Trans. Appl. Supercond., 19 (3), 532 (2009). DOI: 10.1109 /TASC.2009.2019426

[11] F.C. Wellstood, C. Urbina, J. Clarke, Phys. Rev. B, 49 (9), 5942 (1994). DOI: 10.1103/PhysRevB.49.5942

[12] G.L. Pollack, Rev. Mod. Phys., 41 (1), 48 (1969). DOI: $10.1103 /$ RevModPhys.41.48

[13] D. Chouvaev, L. Kuzmin, M. Tarasov, Supercond. Sci. Technol., 12 (11), 985 (1999). DOI: $10.1088 / 0953-2048 / 12 / 11 / 386$

[14] Cadence AWR [Электронный ресурc]. URL: https://www.awr.com/awr-software/products/awr-designenvironment

[15] А.В. Меренков, С.В. Шитов, В.И. Чичков, А.Б. Ермаков, Т.М. Ким, А.В. Устинов, Письма в ЖТФ, 44 (13), 59 (2018). DOI: 10.21883/PJTF.2018.13.46328.17149 [A.V. Merenkov, S.V. Shitov, V.I. Chichkov, A.B. Ermakov, T.M. Kim, A.V. Ustinov, Tech. Phys. Lett., 44 (7), 581 (2018). DOI: $10.1134 / \mathrm{S} 106378501807012 \mathrm{X}$.

[16] С.В. Шитов, Письма в ЖТФ, 37 (19), 88 (2011). [S.V. Shitov, Tech. Phys. Lett., 37 (10), 932 (2011). DOI: $10.1134 / \mathrm{S} 1063785011100117]$.

[17] A.L. Woodcraft, M. Barucci, P.R. Hastings, L. Lolli, V. Martelli, L. Risegari, G. Ventura, Criogenics, 49 (5), 159 (2009). DOI: 10.1016/j.cryogenics.2008.10.024

[18] B.S. Karasik, C.B. McKitterick, T.J. Reck, D.E. Prober, IEEE Trans. Terahertz Sci. Technol., 5 (1), 16 (2015). DOI: 10.1109/TTHZ.2014.2370755 